\title{
Shorter height exacerbates the influence of abdominal fatness on metabolic risk in adults
}

\author{
E. Moqeem and H.D. McCarthy \\ Public Health Nutrition Research Group, London Metropolitan University, Holloway Road, London N7 8DB
}

Metabolic disease is related to excess visceral fat accumulation, hence the measurement of waist circumference (WC) in clinical and public health practice and the use of 'at risk' WC cut-offs. ${ }^{1}$ These cut offs are recommended without reference to an individual's height. Adult height also influences metabolic health but it is unclear whether these two anthropometric measures interact to influence overall disease risk. Thus the objective of this study was to determine if the height of the individual has a role in the prediction of metabolic and cardiovascular risk beyond that of WC.

Data from the Health Survey for England 2013 were used. 3217-4180 Caucasian adults aged between 18 and 75 years were selected for analysis. Waist and height variables were identified and cases were categorised into gender-specific 'high' and 'low' risk waist and taller than average and shorter than average height. HbAlc, total cholesterol, HDL-cholesterol and systolic and diastolic blood pressure were used as markers for metabolic risk. Measures of associations (Spearman's correlation, odds ratio (OR), regression analysis) were applied to examine the effectiveness of WC as well as height in assessing the cardiovascular risk. WC was also categorized using two different (higher and lower) cut off values for the calculation of ORs for WC and height.

WC and height were both significantly but weakly correlated with all metabolic risk factors (WC, $r^{2}$ ranging from -0.44 to 0.34 , $p<0.01$, height $r^{2}$ ranging between -0.14 to $0.12, \mathrm{p}<0.05$ ). WC was positively correlated with all risk factors except HDL, which had an inverse correlation. Height was negatively correlated with all risk factors except blood pressure, which showed a positive correlation. ORs for WC (at the higher cut off) with total cholesterol, HDL, HbAlc and hypertension were found as 1.35 (95\% CI; $1.07-$ $1.71), 3(95 \%$ CI; $1.76-4.34), 2.7$ (95\% CI; $1.19-3.92)$ and $2.1(95 \%$ CI; $1.03-2.94)$ respectively. OR for short height with total cholesterol, HDL, HbAlc and hypertension were found as 1.47 (95\% CI; 1.13-1.79), 0.9 (95\% CI; 0.41-1.68), 1.7 (95\% CI; 0.93-2.40) and $1.6(95 \% \mathrm{CI} ; 1.02-2 \cdot 31)$. In the multiple regression analysis, height was found to be a statistically strong predictor for all the CVD risk factors but WC was not found to be statistically strong predictor for blood pressure (both systolic and diastolic) though it had a significant regressor effect on the rest of the cardiovascular risks.

These results indicate that metabolic risk is closely correlated with both WC and height. ORs for both WC and height indicated that health risks are greater in individuals with high WC and shorter height compared with those with 'healthy' WC and taller height. In conclusion, although WC alone can be used as a reliable measure of metabolic risk, the inclusion of the measurement of height with WC will add value in the prediction of metabolic health risk with attention particularly paid to individuals with a high WC and shorter height.

1. NICE Clinical Guideline 43, Obesity. (2006). 